At Stanford, the criteria are: Gleason score $\leq 6$ with no pattern 4 or 5 , one single core with cancer, linear extent of cancer $\leq 3 \mathrm{~mm}$, and serum PSA is not considered (2). It is controversial whether percentage or linear extent is the best measure.

The follow-up included twice-yearly digital rectal exam and PSA measurements and yearly surveillance biopsy. Tretament was recommended for biopsy progression which was considered whenever Gleason score was $\geq 7,>2$ positive cores, or $>50 \%$ core involvement. The important finding in Ross et al. study was that PSA double time was not significantly associated with subsequent adverse biopsy findings $(\mathrm{p}=0.83)$ and PSA velocity was marginally significant $(\mathrm{p}=0.06)$.

\title{
References
}

1. Bastian PJ, Mangold LA, Epstein JI, Partin AW: Characteristics of insignificant clinical T1c prostate tumors. A contemporary analysis. Cancer. 2004; 101: 2001-5.

2. Noguchi M, Stamey TA, McNeal JE, Yemoto CM: Relationship between systematic biopsies and histological features of 222 radical prostatectomy specimens: lack of prediction of tumor significance for men with nonpalpable prostate cancer. J Urol. 2001; 166: 104-9; discussion 109-10.

\author{
Dr. Athanase Billis \\ Full-Professor of Pathology \\ State University of Campinas, Unicamp \\ Campinas, São Paulo, Brazil \\ E-mail: athanase@fcm.unicamp.br
}

doi: $10.1590 / S 1677-553820100003000021$

\section{The value of mandatory second opinion pathology review of prostate needle biopsy interpreta- tion before radical prostatectomy}

Brimo F, Schultz L, Epstein JI

Department of Pathology, The Johns Hopkins Hospital Medical Institutions, Baltimore, Maryland, USA

J Urol. 2010; 184: 126-30

Purpose: We determined the value of mandatory second opinion pathology review to interpret prostate needle biopsy before radical prostatectomy.

Materials and Methods: In all cases referred to our institution for radical prostatectomy in 1 year we compared pathological parameters in original and reviewed pathology reports, including benign, atypical or malignant diagnosis, final Gleason score, positive core number, core highest cancer percent and perineural invasion or extraprostatic extension. A major Gleason score discrepancy was defined as a change to a different risk category $(6,7$ and $8-10)$. We defined a significant difference in the highest percent of cancer in a core as $30 \%$ or greater.

Results: Of the 855 cases originally diagnosed as prostatic adenocarcinoma cancer was confirmed in 844 $(98.8 \%)$ by needle biopsy and prostatectomy, of which $9(1 \%)$ were atypical and $2(0.2 \%)$ were benign upon review. A major discrepancy in Gleason score was present in 124 cases (14.7\%), of which 57 (46.0\%) were upgraded and $67(54 \%)$ were downgraded. Of cases with a final Gleason score of $6,8.4 \%$ were originally diagnosed as $7(7.8 \%)$ or $8-10(0.6 \%), 21 \%$ with a final score of 7 had an original score of $6(13.2 \%)$ or $8-10$ $(7.8 \%)$ and 21 of $61(34 \%)$ with a score of $8-10$ were originally diagnosed as 7 or less. There were 80 cases $(64.5 \%)$ of disagreement between scores 6 and 7 . Of the 777 cases with the positive core number in each 
report $71(9.1 \%)$ had discrepancies. After review the positive core number was higher in 45 cases $(63.4 \%)$ and lower in $26(36.6 \%)$. We noted a significant difference in the highest cancer percent in a core in 76 of 844 evaluable cases $(9 \%)$ in which cancer was originally underestimated. In 60 of 76 cases (78.9\%) cancer discontinuously involved the core on review. Review revealed perineural invasion in 138 of 844 cases $(16.3 \%)$ that was not originally reported in 37 of 138 (26.8\%). In 4 cases review showed extraprostatic extension on needle biopsy.

Conclusions: Compared to a smaller study more than 10 years ago at our institution the rate of unconfirmed cancer was identical (1.2\%). To our knowledge this is the first study to analyze concordance upon review of the number of positive cores and maximum percent positive in a core (each discrepancy 9\%). In a few cases mandatory second opinion on prostate needle biopsy results in significant differences that may affect therapy.

\section{Editorial Comment}

This article by Brimo et al. emphasizes the importance of a second opinion pathology review of prostate needle biopsy interpretation before radical prostatectomy. It may result in significant differences that may affect therapy. Of the 855 cases originally diagnosed as prostatic adenocarcinoma, cancer was confirmed in 844 $(98.8 \%)$ by needle biopsy and prostatectomy. Therefore, the rate of unconfirmed cancer was $1.2 \%$. Of these unconfirmed cases $1 \%$ were "suspicious but not diagnostic" and $0.2 \%$ were benign.

The most common benign lesion that simulates adenocarcinoma is partial atrophy. The lesion was reported in the periodic literature in 1998 (1). Architecturally, partial atrophy consists of crowded glands often with a disorganized growth pattern. In contrast to complete atrophy, which can typically be diagnosed at scanning magnification owing to the presence of well-formed glands with a very basophilic appearance, partial atrophy has pale cytoplasm lateral to the nuclei giving rise to pale staining glands that more closely mimic cancer. An additional difficulty in distinguishing cancer from partial atrophy is the positivity for alpha-methylacyl coenzyme A racemase (AMACR) in some acini (2-4).

\section{References}

1. Oppenheimer JR, Wills ML, Epstein JI: Partial atrophy in prostate needle cores: another diagnostic pitfall for the surgical pathologist. Am J Surg Pathol. 1998; 22: 440-5.

2. Herawi M, Parwani AV, Irie J, Epstein JI: Small glandular proliferations on needle biopsies: most common benign mimickers of prostatic adenocarcinoma sent in for expert second opinion. Am J Surg Pathol. 2005; 29: 874-80.

3. Wang W, Sun X, Epstein JI: Partial atrophy on prostate needle biopsy cores: a morphologic and immunohistochemical study. Am J Surg Pathol. 2008; 32: 851-7.

4. Worschech A, Meirelles L, Billis A: Expression of AMACR (alpha-methylacyl coenzyme A racemase) in partial and complete focal atrophy on prostate needle biopsies. Anal Quant Cytol Histol. 2009; 31: 424-31.

Dr. Athanase Billis

Full-Professor of Pathology State University of Campinas, Unicamp

Campinas, São Paulo, Brazil

E-mail: athanase@fcm.unicamp.br 